\title{
Una regina invisibile: Elvira di Sicilia (1103-1135)
}

An invisible queen: Elvira of Sicily (1103-1135)

\author{
(4) Glauco Maria Cantarella \\ Alma Mater Studiorum, Università degli Studi di Bologna, Italia \\ cantaglau@gmail.com
}

\section{Riassunto}

Elvira di Castiglia-León, figlia di re Alfonso VI, fu la prima moglie di Ruggero II, conte di Sicilia e re dal 25 dicembre 1130, che sposò intorno al 1117; dunque fu la prima regina di Sicilia. Morì giovane e praticamente non lasciò tracce di sé, ma questo non spiega perché la storiografia se ne sia praticamente disinteressata o abbia cercato interpretazioni talmente generiche da non avere troppa consistenza. Questo saggio si propone di riprendere la questione e di esplorare il contesto della vicenda.

\begin{abstract}
Elvira of Castile-León, daughter of King Alfonso VI, was the first wife of Roger II, count of Sicily and king since 25 December 1130 . She married Roger II circa 1117 and was the first queen of Sicily. Despite having died young and left few traces of herself, this does not explain why historiography has been practically uninterested about her figure, or the generic and inconsistent interpretations about her. This essay aims to address this topic and explore the context of Elvira's life.
\end{abstract}

Elvira di Castiglia León fu la prima moglie di Ruggero II di Sicilia. Era figlia di Alfonso VI e di Isabella. ${ }^{1}$

Isabella si chiamava Zaida; già moglie del mulūk di Cordova e nuora di quello di Siviglia, dopo l'arrivo degli Amoravidi in al-Andalūs era stata presa sotto la protezione di Alfonso VI e ne era divenuta la concubina. Fu battezzata come Isabella dopo la nascita, intorno al 1094, dell'unico figlio maschio di Alfonso, Sancio. O forse fu battezzata di fretta tra il 15 gennaio 1100 (data in cui risulta morta la terza moglie del re, Berta) e il 14 maggio 1100 (quando Isabella appare come già regina).

\section{Parole chiave}

Elvira di Castiglia Ruggero II Sicilia

\section{Keywords}

Elvira of Castile Roger II Sicily

1. Presento qui un'anticipazione, ampiamente rimaneggiata e ampliata, della ricerca che comparirà nel mio Ruggero II, Salerno Editrice, Roma, prev. novembre/dicembre 2020 . 
2. Su Zaida-Isabella cfr. Salazar y Acha (2007, p. 237, p. 239 n. 29, p. 240 n. 30). Cfr. "Sancius puer regis filius quod pater fecit laudo et cf."; Hernández (1985, p. 12), $n^{\circ} 12$ (1103 giugno 22); "Sancius infans regis filius quod pater fecit cf.", Hernández (1985, p. 14), n 14 (Monzón 1107 marzo 8).

3. Per i ritratti femminili Castiñeiras González (2002, p. 193). Fernández Flórez (1991, p. 78), $n^{\circ} 1200$ (1118): “Infantissa domna Sancia, soror regine, conf. Infantissa domna Sancia, filia regine, conf. Infantissa domna Taresia conf. Infantissa domna Geluiria conf."; salvo, naturalmente, che si tratti di una omonimia: ma ritorneremo su questo.

4. Purtroppo non mi è stato possibile consultare Vital Fernández (2019).
Sancio era linfante e come tale compare sempre nella documentazione a partire dal 1103 con le sottoscrizioni sue o apposte a suo nome ("Domnus Sancius, infans quod pater fecit, confirmo"; ancor più chiaro nel 1107, a 13 anni: "Sancius infans, filius eiusdem imperatoris"). Era una garanzia per la successione al trono, finalmente, dopo tante figlie femmine, ma Alfonso non si sentiva abbastanza sicuro, un solo figlio maschio non garantiva abbastanza: e aveva ragione. Nel 1108 Sancio venne istigato a combattere in campo aperto contro gli Almoravidi fuori da Toledo e mandato a morte pressoché certa da una evidentissima cospirazione dei nobili che non fecero nulla per difenderlo come sarebbe stato loro obbligo, a parte il conte García Álvarez, alférez del re cui linfante era stato affidato per essere cresciuto nell'educazione delle armi e che morì nel tentativo di fargli scudo con il suo corpo. Ma su questo dovremo tornare. ${ }^{2}$ Naturalmente Alfonso VI aveva continuato nei suoi tentativi di avere altri figli maschi da Isabella; ma senza fortuna, furono di nuovo figlie femmine.

Nel 1103 nacque Elvira. Il nome assegnato alla bambina la poneva nella linea ufficiale della casa di Pamplona cui apparteneva Alfonso VI e che, ad esempio, venne registrato sontuosamente negli anni ' 30 del sec. XII dalla galleria di ritratti del Tumbo A di Santiago de Compostela. Figlia del re e della regina, era ovviamente una infanta, e come "infantissa" è registrata nella sottoscrizione di un documento relativo a Sahagún nel 1118, quando però era altamente improbabile, se non semplicemente impossibile, che fosse presente, per i motivi che vedremo subito (Castiñeiras González, 2002, pp. 187-196). ${ }^{3}$

Naturalmente Elvira deteneva linfantazgo, dotazione di beni della infanta, come sappiamo che era stato assegnato alla sorellastra maggiore Urraca, nata da Costanza di Borgogna nel 1080, e del quale troviamo la prima menzione nel 1107. Così come l'infantazgo era stato attribuito alla sua omonima zia Elvira, morta nel 1099, che l'aveva trasmesso alla pronipote Sancia (figlia di Urraca e di Raimondo di Borgogna) che le era stata affidata per esserne cresciuta (esserne criada "nel senso nobile del termine") e che consisteva fondamentalmente nelle rendite delle città di Tábara e Wamba e del monastero di San Miguel de l'Escalada, il bellissimo edificio in stile mudéjar che si vede alle porte di León sul camino de Santiago. Del resto l'infantado monastico costituì una caratteristica del regno di León, anzi una sua articolazione, fin dal secolo X e si dissolse solo negli anni '40 del secolo XII (Th. Martin, 2008, pp. 13-14; G. Martin, 2008, p. 25; Henriet, 2008; Reglero de la Fuente, 2018, pp. 419-425).4

Elvira e sua sorella maggiore Sancia lo detenevano proprio perché figlie di Alfonso VI e di "Helisabeth regina" (nel 1107: "Helisabet toletani imperii regina"): così comparivano il 22 luglio 1109, così compaiono l'8 gennaio 1116 come reggenti dellinfantazgo di San Pelagio e San Isidoro a León (ma giustamente Therese Martin ha espresso dubbi sull'efficacia dei loro poteri di reggenti). E sorelle della regina Urraca.

Anche se... La storiografia non comprende Isabella fra le regine ufficiali della storia di Spagna, a giudicare dal fatto che Isabella II fu sovrana nel terribile secolo XIX spagnolo e Isabella I fu la regina dei Re Cattolici che conquistarono Granada il 2 gennaio 1492: diciamo che la moglie di Alfonso VI e madre di Elvira potrebbe essere chiamata Isabella $\mathrm{O} . .$.

E si è fatta molta confusione e c'è stato un gran discutere: chi ha voluto vedere in lei una transpirenaica, chi ha parlato di due Isabelle distinte, e questo a partire dal Tudense nel secolo XIII. Si può dire di più, la confusione era massima a proposito della sua tomba: era a Sahagún fra quelle costruite nel 1286 da Sancio IV quando si pensava che Isabella e Zaida fossero due persone differenti? o a San Isidoro di León, pantheon di Urraca e della sua famiglia ove però le iscrizioni che ne attesterebbero la presenza sono risultate dipendere alla lettera dal Tudense ed essere dunque 
posteriori e apocrife? Insomma, questa Isabella è una regina fantasma, controversa se si vuole, o piuttosto, e paradossalmente viste le non scarse tracce che ha lasciato nella documentazione, una regina invisibile (Salazar y Acha, 2007, pp. 229-231; Hernández, $\left.1985, \mathrm{nn}^{\circ} .12,14\right) \cdot{ }^{5}$

Di Elvira non sappiamo quasi nulla a parte il fatto che, ovviamente, è nata e che probabilmente nel 1117 andò in sposa a Ruggero II conte di Sicilia (e nella documentazione siciliana il suo nome, come vedremo, ricorre solo dopo la sua morte); lultima volta, salvo errore, che il suo nome compare nella documentazione di León è 1’8 gennaio 1116, dopodiché è registrata solo sua sorella Sancia. Il suo destino ha così poco interessato gli storici che perfino in uno dei lavori migliori su Urraca, madre di Alfonso VII, scritto da Therese Martin, grande specialista di queste tematiche, nel 2005, suo marito viene denominato "duca di Sicilia", un discreto errore peraltro replicato da Georges Martin alla fine del 2010 (Th. Martin, 2005, p. 1149, n. 41)... ${ }^{\mathrm{Ma}}$ proprio la vicenda dell'altra Sancia contemporanea, figlia di Urraca, che abbiamo menzionato prima deve porci qualche interrogativo: se questa venne affidata alla prozia Elvira per essere criada nella sua corte, a chi venne affidata la piccola Elvira per la sua educazione? E anche: a chi venne affidata la sorella maggiore di Elvira, immancabilmente chiamata Sancia come abbiamo visto (tutti nomi della legittimità pamplonese)? Intendiamoci, nel 1102/1103 e negli anni immediatamente successivi.

Cioè prima del cataclisma del 1108, la morte di Sancio, e della morte di suo padre Alfonso VI (1109), che aprirono un terribile vuoto di poteri che si precipitarono a riempire Urraca, vedova di Raimondo di Borgogna sposata in fretta e furia a Alfonso Ramírez re d'Aragona (Alfonso el Batallador), la sua sorellastra Teresa, moglie di Enrico conte di Portogallo, il vescovo di Compostela Diego Gelmírez e naturalmente tutte le aristocrazie variamente schierate e interessate a trarre partito dalla situazione: quelle stesse aristocrazie che avevano fatto spallucce di fronte al dolore disperato (di padre, di re) e impotente di Alfonso VI quando gli era stata annunciata la morte di Sancio.

E comunque perché Elvira di León-Castiglia venne maritata nel 1117 con Ruggero II conte di Sicilia? La sua storia è come un caleidoscopio, per usare una felice immagine di un giovane e valentissimo storico, Enrico Veneziani (2019, pp. 45-46, 61): per cercare di avere qualche lume occorre ripercorrere tutto il contesto, e ripercorrendolo le figure che lo animano prendono forme differenti a seconda delle angolazioni. E per cercare di capirne di più dobbiamo andare indietro di nove anni, proprio alla morte di Sancio (1108). Attenzione: sarà solo un paradigma indiziario, di più -in mancanza di documenti ancora ignoti-non si può fare. Ma forse può chiarire un po' il quadro.

Le fonti latine sulla morte di Sancio sono differenti: abbastanza secca quella della Historia Compostellana, di poco posteriore; drammatica, emotiva, empatica quella di Rodrigo Ximénez de Rada (arcivescovo di Toledo, convinto alfiere della primazia della sua sede in Spagna, cancelliere di Alfonso VIII, magister di diritto e grande diplomatico, che diede un contributo fondamentale all'alleanza che nel 1212 portò alla vittoria di Las Navas de Tolosa, nel 1218 nominato legato papale per la cruzada, che tenne un ruolo attivo anche con Ferdinando III: insomma uomo di mondo, di politica e di corte) un secolo più tardi. E lacunose tutte e due. Non soltanto perché Sancio potrebbe non essere morto sul campo di battaglia di Uclés ma forse a Cuenca o altrove, ma soprattutto perché non solo la Historia Compostellana ma nemmeno Ximénez de Rada dedicano una riga per motivare la decisione di far uscire linfante Sancio, unico erede maschio al trono e probabilmente, seppur di poco, ancora minorenne, ad attaccare battaglia contro gli Almoravidi. Ximénez de Rada suggerisce (attenzione, suggerisce solamente) che la preparazione militare di Sancio non era ancora completa, la sua morte è evocata come qualunque morte di qualunque ragazzino sorpreso e terrorizzato:
5. Non è sfuggito alla confusione H. Salvador Martínez (1992, p. 103), che distingue una Isabella "francesa" ripudiata nel 1106, morta nel 1107, sepolta a León (“un indicio más de que murió apartada de la corte de Alfonso VI") da una IsabelZaida, "enterrada en Sahagún junto con todas las demás mujeres legítimas". Un quadro sintetico e preciso in Th. Martin (2011, p. 147); sul loro infantazgo cfr. p. 178; Th. Martin (2016, pp. 115-116). Fernández Catón (1990), n 1327 (1109 luglio 22): "Sancia, filia supra dicti nobilissimi regis, et Elisabet regine, hoc factum domine et sororis mee confirmo. Geloira eiusdem imperatoris et regine filia [...] confirmo"; n 1351 (1116 gennaio 8): "Regnante regina domna Urraka cum filio suo domno Adefonso in Esperie regno. Sancia et Geloira eiusdem regine sororibus, domum Sancti Pelagii regentibus [...] Soror regine Sancia Adefonsi conf. Soror eiusdem Geloira Adefonsi conf.".

6. Per gli appellativi di Urraca cfr. Th. Martin (2005, p. 1145, nn. 34, 35); G. Martin (2010, p. 33, n. 114). 
7. "rex Adelfonsus... senio et grauedine non permissus, misit Garsiam comitem cum filio suo Sancio adhuc puero, et cum eis magnates et milites regni sui [...] Sicut Domino placuit, pars christiana cepit uirtute Arabum infirmari, et in ea parte ubi comes cum puero aderat cepit forcius inualere; cumque quispiam equm, cui infans Sancius insidebat, grauiter uulnerasset, comiti dixit: "Pater, pater, equs cui insideo est percussus". Cui comes: "Prestolare, quia te etiam ferient successiue". Et in continenti cecidit equs qui fuerat sauciatus, et regis filio simul cadente comes descendit et inter se et clipeum puerum, ut potuit, collocauit, cede undique perurgente. Ipse uero cum esset strenuus, et clipeo puerum tutabatur et undique irruentes cedibus repellebat, set pede ictu gladii amputato non potuit amplius sustentari et incubuit super puerum, ut ipse quam puer antea cederetur [...] Rex dolore ineffabili conturbatus talia dixit eis: "Vbi est

filius meus, iocunditas uite mee, solacium senectutis, unicus heres meus?" Ad quod comes Gomicius sic respondit: "Nobis filium, quem exposcitis, non dedistis". Cui ille: "Etsi dedi aliis, consortes prelii et custodie uos adiunxi; et ille, cuius custodie specialiter deputaui,

incubuit et occubuit super eum; uos autem, relicto puero, cur uenistis?" [...] Quanto enim talia dicebantur, tanto amplius rediuiuis singultis memoria filii torquebatur" (Fernández Valverde, 1987, pp. 216217). Per le traduzioni in italiano cfr. Cantarella (2017, pp. 89-94).

8. "Quod cum filio regis, Sacntio scilicet, fuisset auditum, cuius custodie secundum patris imperium Toleti dominium erat commissum, assumpta nobilitate consulum, stipatus probitate et militia uirorum nobilium ad fugandos hostes sue patrie destructores celeriter est profectus" (Falque Rey, 1988, pp. 57-58). e quando un nemico ferì gravemente il cavallo montato dall'infante Sancho, questi disse al conte: "Padre, padre, il mio cavallo è ferito", e il conte gli rispose: “Fermati, feriranno anche te", e in questo momento cadde il cavallo ferito e il conte smontò e pose fra sé e il suo scudo, come poté, il ragazzo, mentre tutt'intorno incalzava la strage. Era un uomo valoroso, proteggeva il ragazzo con lo scudo e respingeva gli assalitori che arrivavano da ogni parte facendone strage, ma un colpo di spada gli amputò un piede e lui non poté più sostenersi in piedi, e si gettò sul ragazzo per essere ammazzato prima di lui.

Padre, padre: il ragazzo è spaventato, non sa che fare... Fermati: scendi da cavallo prima che cadendo ti travolga e ti impedisca di nuoverti...

E dice chiaramente che intorno a Sancio non si strinsero per proteggerlo quelli che avrebbero dovuto farlo tranne, come abbiamo anticipato, il suo aio, il conte García Álvarez ("Garsia Albariz armiger regis" nei documenti toledani in cui ricorrono tanto Isabella come Sancio) cui linfante era stato affidato e che gli fece scudo col suo corpo finché gli fu possibile: non intervenne nessuno? nessuno si era accorto della piega che stava prendendo o aveva già preso la battaglia? tutti si erano dati alla fuga lasciando soli il conte e l'erede al trono? nessuno tentò di sottrarlo ai suoi nemici rinserrandolo in una piccola formazione che a spron battuto lo portasse via dal campo di battaglia e dal pericolo? Difatti questa è l'accusa che Ximénez de Rada fa formulare al disperato re-padre: "turbato da un dolore impossibile a dirsi, disse loro: "Dov'è mio figlio, la gioia della mia vita, la consolazione della mia vecchiaia, l'unico mio erede?" Al che il conte Gómez gli rispose: "Non avete affidato a noi vostro figlio", e il re: "Anche se lo diedi ad altri, vi ho aggiunto come compagni della guerra e della sua custodia; quegli a cui avevo affidato in modo speciale la sua custodia cadde e soccombette sopra di lui: voi perché siete venuti, lasciato lì il ragazzo?"“; tutti cercano di riportarlo alla ragionevolezza (il che, in una situazione del genere, potrebbe suonare persino oltraggioso se non si facesse leva sul codice di comportamento dei re che non possono manifestare emozioni, neppure le più forti), ma più parlavano "più il ricordo del figlio lo tormentava con nuovi singhiozzi". Però notiamolo, alla fin dei conti il dolore e soprattutto l'indignazione e l'ira regia sono espressi sommessamente, come a testimoniare lo stato di totale impotenza di Alfonso: nei confronti della morte com'è ovvio, ma soprattutto nei confronti dei suoi nobili, da cui dipendeva...?

Situazione strana... Ammettiamo pure che si fossero eccitati gli ardori bellici del ragazzino ansioso di manifestare il suo valore virile e comunque certo di poter contare sui suoi nobili (cioè: sui nobili di suo padre), che è quanto dice la Historia Compostellana ("quando a Sancio, figlio del re, alla cui custodia era stata affidata la signoria di Toledo secondo la suprema volontà del padre, giunse questa notizia, raccolta la nobiltà dei conti, circondato dalla probità e dal valore militare dei nobili, partì velocemente a scacciare i nemici distruttori della sua patria"): 8 ma nessuno era intervenuto a cercare di sconsigliarlo e raffrenare la sua ansia di porsi in luce come vero cavaliere e degno erede di suo padre? Neppure il vero signore di Toledo, l'anziano e autorevolissimo Bernardo di Sauvetat che Alfonso VI aveva voluto a tutti i costi sulla sede toledana sfidando anche il papa Gregorio VII, e che dal re aveva avuto carta bianca, aveva scelto i suoi uomini uno ad uno e di certo manteneva a Toledo un controllo molto più forte e profondo di quello nominalmente esercitato da Sancio o anche da sua madre Isabella? E che nel 1103 aveva ottenuto il privilegio che i suoi palazzi fossero considerati alla stessa stregua di quelli reali e che ogni violazione fosse punita come un'ingiuria fatta al re? Notiamo che nel cosiddetto Quirógrafo de la moneda (1107) Bernardo aveva sottoscritto come "arcivescovo dellimpero toledano e legato della Chiesa romana" e la sua firma ricorreva immediatamente dopo quelle di Alfonso, la regina Isabella, il conte Raimondo, Urraca figlia del re, Sancio figlio del re, Enrico conte di Portogallo, Teresa figlia del re: era l'uomo 
più importante del regno dopo i membri della famiglia reale. Don Rodrigo, successore duecentesco di Bernardo, non proferisce una sillaba sul ruolo tenuto dal suo illustre predecessore in quell'occasione: per lui, semplicemente Bernardo non c'è. Il che appare, a dir poco, inverosimile...?

Morto Sancio, costretto Alfonso presidiare il territorio toledano che era rimasto un avamposto leonese (e che, pure, resse benissimo gli urti nei decenni a venire) ${ }_{1}^{10}$ morto Alfonso nel 1109 nella consapevolezza che per il regno si aprivano prospettive burrascose (basta un'occhiata ai sottoscrittori dei documenti reali per rendersi conto del panorama potenzalmente o larvatamente conflittuale quegli anni) e potenzialmente distruttive, ecco la successione: Urraca, figlia di Costanza, diventa regina e convola a nozze con Alfonso d'Aragona: più che un matrimonio una sequenza di deflagrazioni, e certo non solo per via delle preferenze di Alfonso che gli assicurarono il nomignolo di Batallador. All'atto di designazione di Urraca è presente, immancabilmente e in prima fila, Bernardo di Toledo. E Isabella? Le ultime menzioni di Isabella risalgono al 1107 (14 aprile, 14 maggio). Probabilmente morì prima di Alfonso, ma in realtà non lo sappiamo. E la grande e non disinteressata confusione che si fece subito al suo riguardo (come ha scritto con grande e tranchante chiarezza il Salazar y Acha, "las crónicas [...] están casi siempre mediatizadas por el mundo oficial. Su autor es un personaje de algún modo vinculado con el poder y que tiene por tanto que convivir con él. Las crónicas reflejan, de un modo siempre evidente, la ideología, la mentalidad, los prejuicios de su autor o de la persona o institución a quién éste sirve") finì per condurre ad una vera e propria cancellazione della memoria; anche se nel 1115 venne ricordata una volta da Urraca fra le mogli di suo padre (Salazar y Acha, 2007, pp. 241, 235,230 n. 9, 241; Gambra, 1998, n 189, pp. 483-484).

Nel nordovest del regno Enrico di Borgogna, marito di Teresa, in concordia con la moglie e appoggiando ora el Batallador ora Urraca cercava di estendersi verso est, e fra il 1109 e il 1112 (anno della sua morte) occupò Zamora, Astorga, Salamanca, forse anche Ávila e parte dell'Estremadura; basta dare un'occhiata alla carta geografica (ancor meglio: viaggiare per quelle aree) per vedere come puntasse a insignorirsi di un ampio corridoio territoriale nord-sud. Di fatto governava come re. Ma così entrava in competizione con il vescovo di Santiago Diego Gelmírez; Urraca si vide costretta ad affidare il figlio Alfonso (il futuro Alfonso VII) a Gelmírez, tanto per cercarne l'appoggio (in pratica, il bambino era ostaggio del vescovo a garanzia dell'atteggiamento di sua madre la regina) quanto probabilmente per sottrarlo al battagliero marito. Gli arcivescovi di Braga e il vescovo di Coimbra erano già da tempo, almeno dal 1102 , in rotta di collisione con Gelmírez; l'arcivescovo di Braga era, è stato scritto, "un protégé" di Bernardo di Toledo. Che certo non poteva vedere di buon occhio le pretese primaziali di Santiago (già espresse nel 1049 e già rigettate da Roma) e le ambizioni a largo raggio di Gelmírez che investivano sedi galiziane, portoghesi e castigliane (Lugo, Coimbra, Salamanca: è il quadro complicato delle sedi episcopali fra Portogallo e Galizia) ma nemmeno poteva avere in simpatia quelle di Coimbra né di Braga. Aggiungiamo che se Braga nel 1109-1110 ebbe conflitti con Teresa e godeva delle simpatie di Urraca, Teresa sosteneva e continuò a sostenere un uomo molto vicino a Gelmírez, Ugo, sulla sede di Oporto.

Il tutto sotto l'occhio attento e interventista del nuovo abate di Cluny, Pons (Ponzio) de Melguelh, e dei suoi plenipotenziari nella penisola iberica, Dalmazzo e Stefano Juret (Geret) (Amaral y Barroca, 2012; Lay, 2009; Mariani y Renzi, 2019, pp. 67-69, 71-74, 86-87; Cantarella, 2014, pp. 61-91).

In questa situazione magmatica Bernardo si trovava in ottima posizione: non avendo fatto nulla per contrastare la morte di Sancio aveva oggettivamente reso un enorme favore tanto a Teresa quanto a Urraca, ma ora doveva guardarsi dai suoi nemici.
9. Cfr. Hernández (1985, pp. 11-12), n 12. Gambra (1998, pp. 484-485), n' 189 ("Toletani imperii archiepiscopus et Romane ecclesie legatus"). Anche per queste indicazioni ringrazio l'amico e collega Francesco Renzi, della Universidade Catolica di Oporto.

10. Rapido ma efficace, Reilly (1993, pp. 98-99). 
11. Jaffé et al. (1885), 6414 (Laterano, 1114 dicembre 4), 6474, 6475 (Anagni, 1115 novembre 3$)=$ epp. 441, 442, coll. 390C-391C ("Nos autem in his vehementer regni turbationem et aetatis tuae gravedinem infirmitatisque pensamus"); cfr. Cantarella (1997, pp. 179-180; 2014, pp. 388-389); Gordo Molina y Melo Carrasco (2018, pp. 43, 131).

12. Quanto alle presenze di Bernardo cfr. Fernández Flórez (1991), $n^{\circ} 1176$ e seguenti.
E uno dei nemici principali, se non il principale, era proprio Maurizio, già vescovo di Coimbra e dal 1109 arcivescovo di Braga. Il 18 aprile 1114 Maurizio venne sospeso dall'ufficio per "nequizia e disubbidienza (nequitia et inoboedientia: accuse generiche ma colpe gravissime, la inoboedientia equivaleva all'eresia) nei confronti del papa. Era rientrato nel 1108 da un fruttuosissimo pellegrinaggio in Terrasanta, si era trovato in mezzo ai tumulti del regno e aveva rafforzato i suoi legami con i potenti e onnipresenti cluniacensi; nel 1114 si vide costretto a rimettersi in viaggio, si precipitò a Roma, vi giunse forse allinizio dell'autunno, conseguì il ripensamento di Pasquale II che il 4 dicembre gli confermò i confini della sua arcidiocesi. Non solo: undici mesi dopo il papa si pronunciò nuovamente in suo favore ricordando al vecchio Bernardo di Toledo le sue forti preoccupazioni per "il turbamento del regno e la gravezza della tua età e della tua infermità" (insomma, poco ci mancava che evocasse direttamente il rimbambimento senile...): il che ci dice che l'azione contro Maurizio era stata promossa o patrocinata o almeno incoraggiata proprio da Bernardo di Toledo... Maurizio non rientrò mai più nella penisola iberica (e finì per essere l'antipapa di Enrico V come Gregorio VIII); non si sa dove passò il 1115 ma l'azione di Pasquale II può essere un indizio del fatto che seppe tessere preziosi legami a Roma, fino a diventare familiaris del cancelliere Giovanni di Gaeta (relazioni che, paradossalmente, lo condussero a chiudere tanto malamente la sua traiettoria di vita), ma evidentemente -e forse proprio per la sua attività presso la Sede Apostolica- nella penisola iberica continuò ad essere sentito come un pericolo e un anno più tardi, tra il dicembre 1116 e il gennaio 1117, un legato papale (Bosone, cardinale di Sant'Anastasia) percorse Galizia, Castiglia e Portogallo per risolvere le questioni, e infine a febbraio presiedette a Burgos una sinodo che scomunicò Maurizio (Renzi, 2019, pp. 19-44)...11

Toledo. Avamposto del regno ma insieme perno e cuore del regno. Centro focale del nostro caleidoscopio. Proviamo a ruotarlo.

Bastione da cui si potevano vedere con maggior distacco, rispetto al nordest scosso dalle guerre e dai sommovimenti di tipo comunale, le cose del regno. Bernardo, una garanzia per il regno se Alfonso VI l'aveva tanto fortemente voluto. Sancio, mandato a presidiare Toledo proprio per limportanza, non solo strategica ma simbolica, che a Toledo si annetteva. Bernardo, costantemente presente nella documentazione al fianco della regina. Anche il dodicenne Alfonso VII, nel 1117, fu mandato a Toledo. Sancio a Toledo... Perché non anche le sorelline, le infantas di Alfonso VI e di Isabella, regina dellimpero toledano? dove avrebbero potuto essere più sicure che sotto la protezione di Bernardo? dove avrebbero potuto avere una preparazione migliore se non nella sede che custodiva il maggior apparato di memoria documentaria dell.Iberia cristiana? Soprattutto dove, se non a Toledo, si sarebbe potuto scrutare con maggior attenzione l'orizzonte per maritarle vantaggiosamente per il regno? E tanto più negli anni dei, chiamiamoli così, disordini matrimoniali di Urraca con Alfonso d'Aragona; il 22 gennaio 1113 si sottoscriveva come "Regnante regina Horracha, cum suo filio, in Toleto et in Legione" (Fernández Flórez, 1991, n 1187). ${ }^{12}$

Facciamo un altro giro del caleidoscopio, altri tasselli intervengono a cambiare del tutto il quadro ma senza discostarsi dal nucleo focale.

Sancia verrà sposata nel 1120 al conte di Lara, un fedelissimo di Urraca. Nel 1120 Elvira è già madre e fuori dalla Spagna, e proprio intorno a quell’anno dà alla luce il suo terzogenito, il bambino viene chiamato Alfonso. Non è certo per ricordare affettivamente la felice memoria del nonno... o almeno (non volendo né potendo certo negare la componente emotiva degli esseri umani, anche quelli potenti, dei secoli passati) non è solo per quello; dopo di lui nacque Enrico, e con quest’ultimo fu ripresa l'onomastica aleramica. 
Alfonso di Sicilia, che in Spagna avrebbe potuto essere chiamato forse Alfonso Rogéliez, è il terzo Alfonso di quei decenni, con Alfonso Raimúndez, il figlio di Urraca nato nel 1105, e Alfonso Enríquez (o Afonso Enríques, come si preferisce), il figlio di Teresa nato nel 1109. Tre sorelle, tre figli, tre rivendicazioni di legittimità. Ma attenzione: sotto il profilo strettamente dinastico solo il Raimúndez e il Rogéliez (chiamiamolo così, sempre per chiarire meglio lo stato delle cose) avrebbero potuto dirsi perfettamente legittimi, perché Teresa, per quanto legittimata da suo padre, era figlia di una concubina, Jiména Muñoz; quindi la partita della legittimità si giocava soltanto fra tre donne e i loro figli, Urraca ("Dei gratia regina et imperatrix Yspanie": 1110; "regina, piisimi Aldefonsi tocius Yspanie imperatoris filia": 1125); Sancia ed Elvira... naturalmente nel caso che Urraca e suo figlio fossero venuti a mancare per qualche non imprevedibile motivo, malattia, incidente (anche se non del tipo di quello occorso a Sancio, va da sé): chi avrebbe mai potuto escludere una eventualità simile? ${ }^{13}$

Ma questo si sapeva già prima che Elvira fosse in età di generare...

Lo sapeva sicuramente anche Elvira da quando ebbe l'età per capire ed essere educata; $\mathrm{e}$ lo sapeva anche suo marito, o al terzogenito non si sarebbe imposto un nome estraneo alla linea degli Altavilla... Allora, sposandola a quasi $2000 \mathrm{~km}$ di distanza con un ricco conte che aveva già 22 anni, stava presidiando con la sua flotta il Canale di Sicilia e le rotte est-ovest del Mediterraneo, poteva avere dinanzi a sé un grande futuro e poteva già vantare di essere figlio di una regina (Adelaide, dal 1113 regina di Gerusalemme) non soltanto le si procurava un matrimonio all'altezza del suo rango (figlia di regina, sorella di regina regnante) ma soprattutto si liberava il regno, già tanto magmatico, dalla sua presenza e la si eliminava dalla stessa eventualità della competizione.

La sterilizzazione delle infantas di Alfonso VI e di Isabella fu perfezionata con il matrimonio di Sancia, che ricadeva a tutto vantaggio di Urraca visto che il suo fedelissimo conte di Lára, onorato con una infanta sorella della regina, non avrebbe avuto motivo di opporsi: era Sancia che veniva costretta ad una mésalliance, anche se, ricordiamolo, nel secolo XII il lignaggio era un portato delle donne.

Allora... ruotando il caleidoscopio l'immagine che finisce per formarsi di Elvira è diversa da quella che è stata trasmessa finora dalla storiografia: sposata a Ruggero II non solo e non tanto perché "in virtù della sua origine [...] era... adatta a patrocinare presso suo marito una pacifica convivenza tra cristiani e musulmani"; non guardando "verso l'Occidente estremo della riscossa anti-islamica"; non per legare il Normanno di Sicilia "a contesti in dialettica acculturazione con il mondo musulmano" o perché Ruggero "intendesse adottare una politica ispirata a quella del suocero, detto dai musulmani 'l'imperatore di due religioni' " (Houben, 1999, p. 48; Tocco, 2011, p. 53; Tocco, 2017, pp. 212-218; Sciascia, 2019, p. 15): ipotesi intriganti, senza dubbio - cosa ci sarebbe di più suggestivo? Peccato che le cose siano più banali: leelisione dal quadro iberico di una pericolosa concorrente di Urraca. E Urraca torna al centro.

E tornano al centro gli anni delle maggiori turbolenze in León-Castiglia: perché il matrimonio con Ruggero di sicuro è stato negoziato prima del 1115/1116 e perfezionato tra gennaio e giugno di quell'anno (1,8 gennaio Elvira sottoscriveva un documento del vescovo di León, dal 16 giugno comparirà solo la sorella Sancia - prescindendo dalla inverosimile sottoscrizione di Sahagún del 1118 cui abbiamo fatto cenno prima, che presupporrebbe un davvero improbabile ritorno di Elvira in Spagna in prossimità del suo primo parto... e che comunque è importante perché registra le posizioni istituzionali dellintera casa reale, tutta al femminile a parte Alfonso VII); era necessario tempo per combinare questi matrimoni, mesi di viaggi e di trattative: sembra strano doverlo ricordare, ma fortunatamente la storiografia lha fatto, seppure in linea generalissima, nel 2007 ... E nel caso di Ruggero II è semplicemente inverosimile che sua madre Adelaide,
13. Per gli appellativi di Urraca cfr. Th. Martin (2005, p. 1145, nn. 34, 35). Ovviamente a prescindere da quanto ha scritto Salvador Martínez (1992, p. 100, n. 1): "Alfonso tenía otras dos hijas legítimas menores (Sancha y Elvira), habidas en su cuarta esposa, doña Isabel, a las que ni siquiera se menciona en los documentos cuando se discute el problema de la sucesión"; sia perché è evidente che a suo giudizio sarebbero state figlie della Isabella "francesa", sia perché la successione non poteva essere in discussione, con Urraca già maggiorenne... 
14. "Infantissa domna Sancia, soror regine, conf. Infantissa domna Sancia, filia regine, conf. Infantissa domna Taresia conf. Infantissa domna Geluiria conf." (Fernández Flórez, 1991, n 1200).

Dato che, salvo errore, non si conoscono altre contemporanee infante chiamate Elvira non può che trattarsi della moglie del conte di Sicilia: della quale comunque, come per sua sorella Sancia e anche per Teresa del Portogallo, viene registrato lo status istituzionale: infanta era e infanta continuava ad essere, con piena legittimità (Salazar y Acha, 2007, p. 238)... che era non in Sicilia ma dal 1113 a Gerusalemme ove restò fino ai primi mesi del 1117, si fosse lasciata tenere alloscuro della trattativa matrimoniale, quindi occorre forse raddoppiare i tempi di spostamento, Spagna-Sicilia-Terrasanta e viceversa. Anche perché non era così semplice raggiungere la Castiglia e il León, e meno ancora, nel caso, Toledo: via terra, occorreva passare per l'Aragona del Battagliero, e si trattava di un affare complicato come sappiamo ad esempio dalle narrazioni della Historia Compostellana; e via mare non c'era molta scelta se non arrivare a Barcellona o a Tortosa, esponendosi ai rischi dei corsari delle Baleari e insieme dei catalani e dei pisani loro alleati... ${ }^{14}$

A questo punto, visto che in quegli anni il tutto ridondava a vantaggio di Urraca, sarebbe così azzardato avanzare la congettura che uno degli attori principali, se non si vuol dire proprio l'artefice o almeno lideatore, della manovra (o delle manovre, se includiamo il successivo matrimonio di Sancia) fosse Bernardo di Toledo? Alle prese, a sua volta, con le intemperanze egemoniche dellarcivescovo di Braga e del vescovo di Santiago, mine vaganti che (ognuno a suo modo: il primo, per i suoi sforzi di costruire uno spazio ampio e incontrastato di signoria intorno a Compostella godendo anche dei proventi economici che derivavano dal pellegrinaggio alla tomba dell'Apostolo; il secondo, perché la sua azione avrebbe potuto inquadrarsi organicamente in quella signoria che si era costituita sotto il dominio personale di Enrico, tanto più approfittando delle tensioni crescenti fra le aristocrazie e Teresa e il suo amante -e probabilmente secondo marito- Fernando Pérez de Traba, conte di Galizia: insomma, Maurizio avrebbe potuto replicare tra Portogallo, Castiglia e Estremadura proprio quanto Gelmírez stava facendo tra León, Galizia e Portogallo) stavano dando filo da torcere proprio ad Urraca... Che non per nulla nel 1116 fece il clamoroso gesto di amicizia nei confronti di Gelmírez donando a Compostella il prezioso furto sacro di Maurizio di Braga, la testa di san Giacomo: che era stata depositata da Maurizio a Carrión de los Condes, priorato cluniacense che in quegli anni fungeva da vera e propria cassa continua (per dire così) di Cluny, dove Gelmírez depositava le imponenti somme destinate a promuovere le sue ambizioni archiepiscopali a Roma, e presso il quale Maurizio di Braga aveva lasciato la preziosissima tavola di san Basilio (che da lì prese la via di Cluny) e, ben più preziosa, la testa dell'Apostolo; Urraca la fece trasferire a San Isidoro di León, sua creatura, e provvide a donarla a Gelmírez. Aggiungiamo, per gettare un'altra lama di luce sulle turbolenze di quel periodo e di quellarea, il caso di Sahagún, monastero cluniacense teatro di un tentativo comunale dei burgenses fra il 1110 e il 1116 in cui si erano inseriti Alfonso d'Aragona e suo fratello Ramiro il Monaco (che era cresciuto nello stesso monastero in cui era stato formato il contemporaneo abate di Cluny, Pons de Melguelh), e dal quale proveniva lo stesso Bernardo di Sauvetat arcivescovo di Toledo.

Erano nel mirino tanto il regno di Urraca e Alfonso VII quanto Toledo; Bernardo sposando la causa della regina forniva a lei un preziosissimo e autorevolissimo alleato, e metteva se stesso al riparo dalle difficoltà che potevano provenirgli da ovest, dal Portogallo... Una rete di contatti tanto precoce con la Sicilia, che magari poteva essere facilitata dal nutrito corpo di ecclesiastici che sappiamo agire intorno a Ruggero negli anni in cui Adelaide era a Gerusalemme, potrebbe anche dar ragione di quanto ha osservato Francesco Renzi nel 2018 sugli stretti rapporti fra Ximénez de Rada e la Cronaca di Romualdo di Salerno (Takayama, 1993, p. 51; Mariani y Renzi, 2019, pp. 69-70; Renzi, 2018, pp. 227-231; Cantarella, 1997, pp. 156-157).

Ovviamente sono solo congetture. Come sarebbe una labilissima congettura ipotizzare che il collegamento fra la casa comitale di Sicilia e la casa reale di León Castiglia si dovesse all'opera degli onnipresenti cluniacensi, che in Sicilia compaiono in una data imprecisata e imprecisabile fra il 1110 e il 1119 (anche se, coincidenza curiosa, lipotesi prevalente nel secolo scorso additava lanno 1114) con la fondazione a Sciacca da parte di Jullita (Giuditta), sorella (sorellastra) di Ruggero II, del monastero di Santa 
Maria delle Giummare; un'operazione episodica ancorché destinata a durare nel lungo periodo, ma che si colloca nell'età della Cluny trionfante. E' vero che nel giro di una decina d'anni Cluny verrà travolta dalla più grave crisi della sua storia secolare; ma nel 1110-1119 quanto sarebbe accaduto di lì a poco non era neppure immaginabile... ${ }^{15}$

Un'altra rotazione del caleidoscopio, e si compone la figura di Alfonso, figlio di Elvira e di Ruggero. Che seppure ben conosciuto dalle fonti narrative del regno di Sicilia come cavaliere elegante e valente morto troppo giovane (1144), duca di Napoli e principe di Capua, non compare mai nella documentazione ufficiale in quanto tale: cioè, non compare mai come Alfonso ma come Amphussus, Anfusus, Amphusus, Amphulsus. Curiosa deformazione del nome, che ricorda un po' quella che di Alfonso suo nonno viene fatta nel Roman de Brut, "Amfurcio"; un po' meno curiosa se si pensa al fatto che la documentazione castigliana registrava Alfonso VI come Adefonsus. Ma che per quanto riguarda la storia del regno in Spagna ne fa un fantasma in più. ${ }^{16}$

Elvira morì il 6 febbraio 1135. A 32 anni, seppure li aveva già compiuti. Di che cosa morì non sappiamo. Visto come Alessandro di Telese, cronista ufficiale dei trionfi di Ruggero II nella penisola, dispone la successione dei tempi nella sua narrazione, sembra più probabile una malattia infettiva, magari la medesima contratta dal marito che fece disperare per la sua vita, piuttosto che "un male incurabile" di cui avrebbe sofferto "da qualche tempo".

Elvira, una regina invisibile. Senza storia, senza volto, meno di un'ombra. Il suo stato di invisibilità è sottolineato dal fatto che il suo nome inizia a comparire solo dopo la sua morte e in due sole occasioni, i diplomi di Ruggero II del 1140 e del 1142; da quello del 1140 sappiamo che "la regina Elvira di buona memoria" aveva una cappella abbondantemente dotata, evidentemente per la commemorazione liturgica visto che si trattava di pertinenze della chiesa cimiteriale di San Giorgio. In più ci sono quattro documenti falsi... uno contraffatto scandalosamente, visto che il falsario non si è nemmeno accorto che l'aveva datato "Palermo, 6 maggio 1550".

Di lei sappiamo soltanto che è esistita. Come un'altra sua figlia, di cui ignoriamo anche il nome: forse si chiamava Adelaide come la nonna paterna. E comunque persino questo, la memoria nellinvisibilità, è un privilegio che la storia ha riservato a pochissimi. Ma almeno non è stata colpita dalla damnatio memoriae come sua madre... ${ }^{17}$
15. Cfr. Cantarella (1978, pp. 113-114; 2020); cfr. Houben (1996, pp. 17-18), che oscilla curiosamente fra 1103 e $1134 / 35$, seppur con un lapsus calami che parla di "carta del 1103"; ma altrettanto significativamente pone questa fondazione in relazione proprio con Elvira proponendo un suo intervento diretto (pp. 19-20). Recentissima e ottima l'ampia riconsiderazione del problema di Russo (2019).

16. Brühl (1987, p. 138), nº 48 , Palermo 1140 aprile $28 ; n^{\circ} 53$, Ariano 1142 giugno (p. 149); Append. II n ${ }^{\circ}$, [Palermo] 1142 maggio 10 (p. 266). Siragusa (1897, p. 6). Cfr. Ruano (2004, p. 216).

17. De Nava (1991, p. 59); Garufi (1935, p. 222); ad a. 1149 (p. 230 e nn. 5, 6), cfr. Houben (1996, p. 88). Brühl (1987, p. 137), $n^{\circ} 48$ (Palermo 1140 aprile 28); $n^{\circ} 53$ (Ariano 1142 luglio) (p. 149). Falsi: $\mathrm{nn}^{\circ} \dagger 45$, $\uparrow 52$, †76, Append. I/†8 (p. 255). L'ipotesi della malattia infettiva in Houben (1999, p. 86) (ripresa dal Tocco, 2011, pp. 85-86); quella del male incurabile (che comunque niente nelle fonti autorizza a sostenere - a meno che non si voglia usare il termine nel senso strettamente etimologico: che non si poté curare) in Aubé (2016, p. 268). 


\section{Q Bibliografía}

»Amaral, L. C. e Barroca, M. J. (2012). A Condessa-Rainha: D. Teresa. Lisboa: Círculo de Leitores.

"Aubé, P. (2016). Roger Il de Sicile, Paris: Perrin.

" Benito Ruano, E. (2004). ¿Galicia por Guillermo el Conquistador? Anuario de Estudios Atlánticos, 50, 213-217.

» Brühl, C. (Ed.). (1987). Rogerii Il. regis Diplomata Latina (Codex Diplomaticus Regni Siciliae. II. 1). Köln-Wien: Böhlau Verlag.

»Cantarella, G. M. (1978). Osservazioni a proposito di un monastero cluniacense in Sicilia. Benedictina, 25, 109-126.

" Cantarella, G. M. (1997). Pasquale Il e il suo tempo. Napoli: Liguori.

»Cantarella, G. M. (2014a). 'Come in uno specchio'? Di nuovo su Ponzio di Cluny (11091122/26). BISIME, 116, 61-91.

"Cantarella, G. M. (2014b). I Normanni e la Chiesa di Roma. In Chiese locali e chiese regionali nell'alto medioevo, Spoleto 4-9 aprile 2013 (377-406). Spoleto: CISAM.

"Cantarella, G. M. (2017). Imprevisti e altre catastrofi. Perché la storia è andata come è andata. Torino: Einaudi.

»Cantarella, G. M. (Ed.) (2020). Cluny. In I castelli della preghiera. II monachesimo nel pieno medioevo (secoli X-XII). Roma: Carocci (in corso di stampa).

»Castiñeiras González, M. (2002). Poder, Memoria y olvido: la galéria de retratos régios en el Tumbo A de la catedral de Santiago (1129-1134). Quintana: revista de estudios do Departamento de Historia da Arte, 1 (1), 187-196.

» De Nava, L. (Ed.). (1991). Alexandri Telesini abbatis Ystoria Rogerii regis Sicilie Calabrie atque Apulie. Roma: Instituto Palazzo Borromini.

" Falque Rey, E. (Ed.). (1988). Historia Compostellana. Turnhout: Brepols.

» Fernández Catón, J. M. (1990). Colección documental del archivo de la Catedral de León (775-1230), V (1109-1187). León: Centro de Estudios e Investigación "San Isidoro".

» Fernández Flórez, J. A. (1991). Colección diplomática del monasterio de Sahagún (857-1300), IV (1110-1199). León: Centro de Estudios e Investigación "San Isidoro".

» Fernández Valverde, J. (Ed.). (1987). Rodericus Ximenius de Rada. Historia de Rebus Hispanie sive Historia Gothica. Turnhout: Brepols.

" Gambra, A. (1998). Alfonso VI: cancillería, curia e imperio. Il: Colección diplomática. León: Centro de Estudios e Investigación "San Isidoro".

»Garufi, C. A. (Ed.). (1935). Romualdi Salernitani Chronicon: A.m. 130 - A.C. 1178. Bologna: Zanichelli.

» Gordo, A. G. e Melo Carrasco, D. (2018). La reina Urraca I (1109-1126). La práctica del concepto de imperium legionense en la primera mitad del siglo XII. Gijón: Ediciones Trea.

" Henriet, P. (2008). Infantes, Infantaticum. Remarques introductives. e-Spania [En ligne], 5 (juin). https://doi.org/10.4000/e-spania.12593.

» Hernández, F. J. (1985). Los cartularios de Toledo. Catalogo documental. Madrid: Fundación Ramón Areces. 
» Houben, H. (1996). Mezzogiorno normanno-svevo. Monasteri e castelli, ebrei e musulmani. Napoli: Liguori.

» Houben, H. (1999). Ruggero II di Sicilia. Un sovrano tra Oriente e Occidente. Roma-Bari: Laterza.

» Jaffé, Ph., Loewenfeld. S., Wattenbach, W., Kalterbrunner, F. e Ewald, P. (Eds). (1885). Regesta Pontificum Romanorum ab condita ecclesia ad annum post Christum natum 1198, I. Leipzig.

» Lay, S. (2009). The Reconquest Kings of Portugal. Political and cultural Reorientation on the medieval Frontier. New York: Palgrave Mcmillan.

» Mariani, A. y Renzi, F. (2019). Redescubrir un obispo ibérico del siglo XII: Hugo de Oporto y el contexto político-eclesiástico del Condado de Portugal (1112-1136). In A. V. Neyra y V. C. Gerhold (Eds.). Sociedad, cultura y religión en la Plena Edad Media (siglos IX al XIII) (63-93). Buenos Aires: IMHICIHU-CONICET.

» Martin, G. (2008). Le testament d’Elvire (Tábara, 1099). e-Spania [En ligne], 5 (juin). https://doi.org/10.400o/e-spania.8613.

» Martin, G. (2020). Hilando un reinado. Alfonso VI y las mujeres. e-Spania [En ligne], 10 (décembre). https://doi.org/10.400o/e-spania.20134.

» Martin, Th. (2005). The Art of a Reigning Queen as Dynastic Propaganda in Twelfth-Century Spain. Speculum, 8o, 1134-1171.

» Martin, Th. (2008). Hacia una clarificación del infantazgo en tiempos de la reina Urraca y de su hija la infanta Sancha (ca. 1107-1159). e-Spania [En ligne], 5 (juin), https://doi. org/10.400o/e-spania.12163.

» Martin, Th. (2011). Mujeres, hermanas e hijas: el mecenazgo femenino en la familia de Alfonso Vl. Anales de Historia del Arte, Volumen Extraordinario (2), 147-179.

» Martin, Th. (2016). Fuentes de Potestad para reinas e infantas: el Infantazgo en los siglos centrales de la Edad Media. Anuario de Estudios Medievales, 46 (1), 97-136.

»Reglero de la Fuente, C. (2018). El infantado monástico: del espacio a la memoria. In F. Arias Guillén y P. Martínez Sopena (Eds.), Los espacios del rey. Poder y territorio en las monarquías hispánicas (siglos XII-XIV) (419-436). Bilbao: Universidad del País Vasco-Euskal Herrico Uniberstatea.

» Reilly, B. F. (1993). The Medieval Spains. Cambridge: Cambridge University Press.

» Renzi, F. (2018). 'Imperator Burdinum Hispanum Romanae sedi violenter imposuit'. A Research Proposal on the Archbishop of Braga and Antipope Gregory VIII, Maurice 'Bourdin'. Imago Temporis. Medium Aevum, XII, 211-235.

» Renzi, F. (2019). Da Coimbra a Costantinopoli fino a Cluny. Il viaggio di Maurizio "Burdino" e la donazione della Croce all'abate Ponzio di Melgueil (1104-1120). In M. Ferrero (Ed.), Un abate, un monastero, un Crocifisso. Ponzio di Melgueil da Cluny a Campus Sion, Atti del convegno (Bassano del Grappa, 2 Giugno 2018) (19-44). Vicenza: Scriptorium.

» Russo, M. A. (2019). Sulle origini del priorato cluniacense di Santa Maria delle Giummare di Sciacca (XII secolo). RiMe. Rivista dell'Istituto di Storia dell'Europa Mediterranea, 4 (II), 5-30.

»Salazar y Acha, J. (2007). De nuevo sobre la mora Zaida. Hidalguía: la revista de genealogía, nobleza y armas, 231, 225-242.

»Salvador Martínez, H. (1992). La rebelión de los burgos. Crisis de estado y coyuntura social. Madrid: Tecnos.

»Sciascia, L. (2019). Tutte le donne del Reame. Regine, dame, pedine e avventuriere nella 
Sicilia medievale. Palermo: Palermo University Press.

» Siragusa, G. B. (Ed). (1897). La Historia o Liber de regno Siciliae e la Epistola ad Petrum Panormitane Ecclesie Thesaurarium di Ugo Falcando. Roma.

» Takayama, H. (1993). The Administration of the Norman Kingdom of Sicily. Leiden-New York-Köln: Brill.

» Tocco, F. P. (2011). Ruggero II: il drago d'Occidente. Palermo: Flaccovio.

» Tocco, F. P. (2017). Ruggero II, re di Sicilia. In AA.VV. Dizionario Biografico degli Italiani, vol. 89 (212-218). Roma: Istituto dell’Enciclopedia italiana.

» Veneziani, E. (2019). La caduta di Ponzio, dramatis personae. In M. Ferrero (Ed.), Un abate, un monastero, un Crocifisso. Ponzio di Melgueil da Cluny a Campus Sion, Atti del convegno (Bassano del Grappa, 2 Giugno 2018) (45-61). Vicenza: Scriptorium.

» Vital Fernández, S. (2019). Alfonso VII de León y Castilla (1126-1157). Las relaciones de poder en el centro de la acción política y social del Imperator Hispaniae. Gijón: Ediciones Trea. 